\title{
Evolution of the large-scale magnetic field over three solar cycles
}

\author{
J. Todd Hoeksema \\ Hansen Experimental Physics Laboratory, Stanford University \\ Cypress C-13, 466 Via Ortega, Stanford, CA 94305 USA \\ email: JTHoeksema@Solar.Stanford.edu
}

\begin{abstract}
Observations of the large-scale magnetic field show that the current extended solar cycle minimum is different from the three previous well-observed minima. The weaker polar fields increase the relative influence of middle and low-latitude flux patterns on the configuration of the corona and heliosphere. A much larger portion of the open flux originates in equatorial coronal holes. Even though the mean magnetic field of the Sun as a star is the weakest since measurements began, the sector structure of the interplanetary field, though smaller in magnitude, reached fairly high latitude until 2009. The emergence of active regions through the cycle and transport of flux from low to high latitudes also show quite different patterns, providing insight into the dynamo that drives the cycle. Long records of synoptic observations provide a rich source of information about solar activity that must be maintained.
\end{abstract}

Keywords. Sun: activity; Sun: magnetic field; Stars: activity.

\section{Introduction}

The Sun's magnetic field has been well measured since the mid 1970s. The 22-year magnetic cycle that drives the 11-year sunspot periodicity originates deep in the interior, near the base of the convection zone. The Sun's dynamo, driven by convection, differential rotation, and meridional flow, provides an accessible example of how magnetic field generation occurs in stars. The solar cycle is dominated by localized eruption of active regions that emerge in a systematic way over time to produce the classic butterfly diagram, where sunspots appears at middle latitudes early in the cycle and the activity belt gradually moves equatorward. The weaker large-scale photospheric flux patterns are the consequence of the emergence, transport, and interactions of active regions and the ubiquitous short-lived small-scale fields generated closer to the surface. The essentially unipolar polar caps that reverse near the maximum of each cycle are the most prominent features of the large-scale field. The minima in 1976, 1986, and 1996 were quite similar, in the sense that the magnetic field was relatively weak except at the poles. This affected the configuration of the coronal and heliospheric field as well. The situation changed as Cycle 23 slowly came to an end, with the polar field weaker by about $40 \%$, the equatorial patterns more prominent, and the duration of the cycle much longer.

Section 2 presents some of the global properties of the three previous solar cycles. In section 3 the consequences for the heliosphere are described. The origin of the large-scale patterns in terms of the zonal characteristics is discussed in section 4 .

\section{Global Properties of the Solar Magnetic Field}

Qualitative and indirect measures of solar activity, such as the sunspot number, geomagnetic activity, and changes in atmospheric isotopic abundance, extend relatively 
far back in time and give a valuable perspective on the variability of the cycle. Direct measures of the synoptic magnetic field provide a record that extends back less than four solar cycles. Figure 1 shows a measure of the large-scale photospheric magnetic field made at the Wilcox Solar Observatory at Stanford. The instrument has been stable, so the values over the 34-year period are consistent, though not absolutely calibrated. Clearly, the polar regions reverse sign each cycle at or shortly after maximum. The reversal does not necessarily take place at the same time north and south. There is a strong annual periodicity in each hemisphere due to the variable viewing angle caused by the inclination of the ecliptic. The variation suggests that the polar field is strongly peaked [Svalgaard et al., 1978]. The total flux above 55 degrees is often not balanced between the two hemispheres; note for example the strong asymmetry in 1994. The polar field evolves slowly in response to the arrival of flux from lower latitudes (see section 4). The average polar field strength is much weaker during the current minimum, about $0.6 \mathrm{G}$ in 2008, compared to the three previous minima: 1.0 in 1976, 1.3 in 1986, and 1.1 in 1996.

Similar features appear in the dipole field. The synoptic magnetic field can for each solar rotation be decomposed into harmonic components at the photosphere. The center panel of Figure 2 shows that the axial dipole strongly resembles the polar field aperture measurements shown in Figure 1. At the minima the axial dipole varies only a little for an extended interval. The peak strength is reached a year or two after the reversal that occurs near maximum. The total dipole is dominated by the axial component near minimum. However, during the maximum phase the equatorial component is often larger. The configuration of active regions and remnants determines the equatorial component. The patterns emerge and decay with a typical time scale of 12 to 18 months. The peaks can be quite large, for example in 1982 and 1991, but are short lived. The maximum in the total dipole coincides with these equatorial peaks. The minimum in the dipole

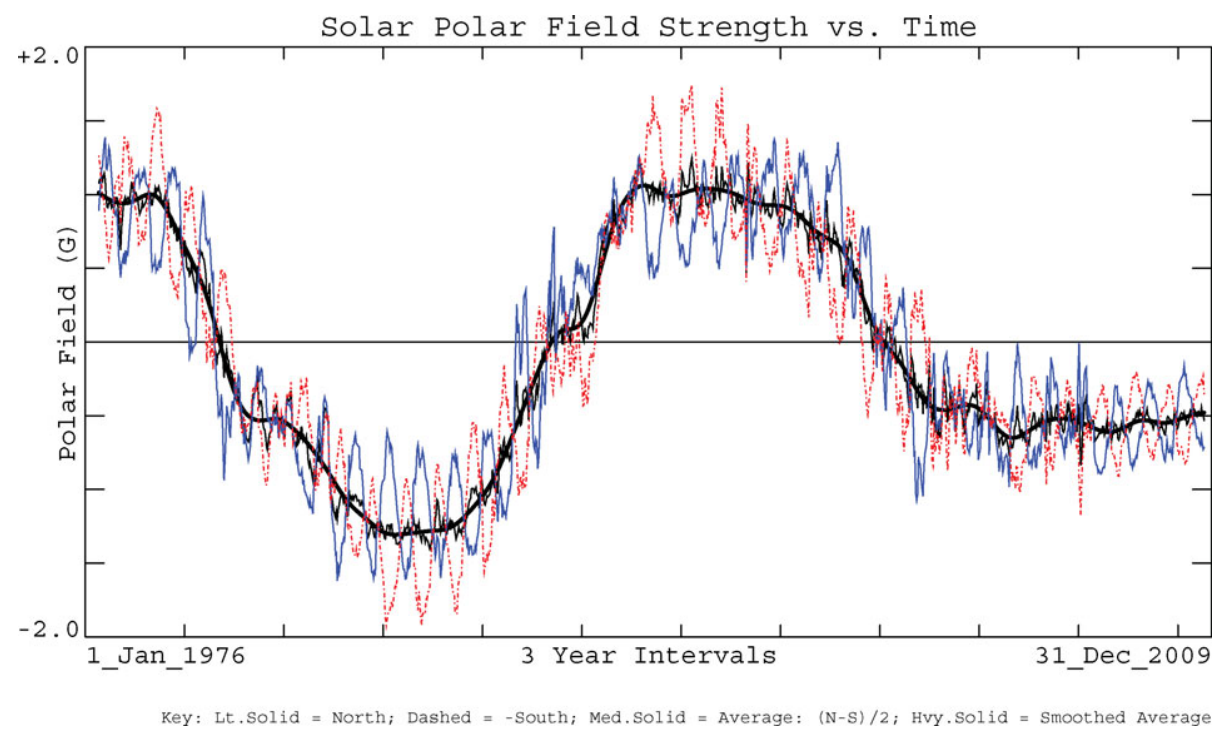

Figure 1. The graph show the polar field strength measured in a small 3 arc-minute aperture at the poles during the interval 1976-2009. The light solid and dashed lines show the 30-day average of the line-of-sight component of northern field and the negative of the south above 55 degrees, respectively. The medium solid line is the average and the heavy solid line is that curve filtered to remove any annual periodicity due to the inclination of the ecliptic. Polar reversals occurred in 1980, 1990, and 2000 in association with the maximum of the cycle. The polar fields build to a long maximum during the declining phase of the cycle. 
magnitude also occurs around the maximum of the solar cycle when the slowly varying polar field is near zero and the variable equatorial component has a minimum. Like the sunspot number, the axial dipole is much weaker during the current solar minimum and the duration of the minimum is much longer. The current value is about $-0.26 \mathrm{G}$ compared with values of $0.6,-0.7$, and 0.55 in the minimum years 1976, 1986, and 1996 .

The reduced value of the polar cap field changes the configuration of the large-scale field in the overlying corona and heliosphere, especially at minimum. It may also presage a smaller maximum for Solar Cycle 24 .

\section{The Effect of the Polar Field on the Corona and Heliosphere}

The complexity of the photospheric field pattern simplifies in the overlying corona and the heliosphere. Coronagraph observations at various heights show this, as do in situ measurements of solar wind structure throughout the heliosphere. One convenient way to model the large-scale structure of the solar atmosphere during the solar cycle is to use a potential field approximation applied to the photospheric field in conjunction with an imposed source surface located at some height at which the field becomes radial and no longer changes with height, [e.g. Schatten, 1968 and Hoeksema et al., 1982]. Of course much more sophisticated force-free field and MHD models can be applied to calculate more quantities, predict events, refine the details, and investigate time variations, but the basic large-scale configuration of the heliospheric field remains the same.

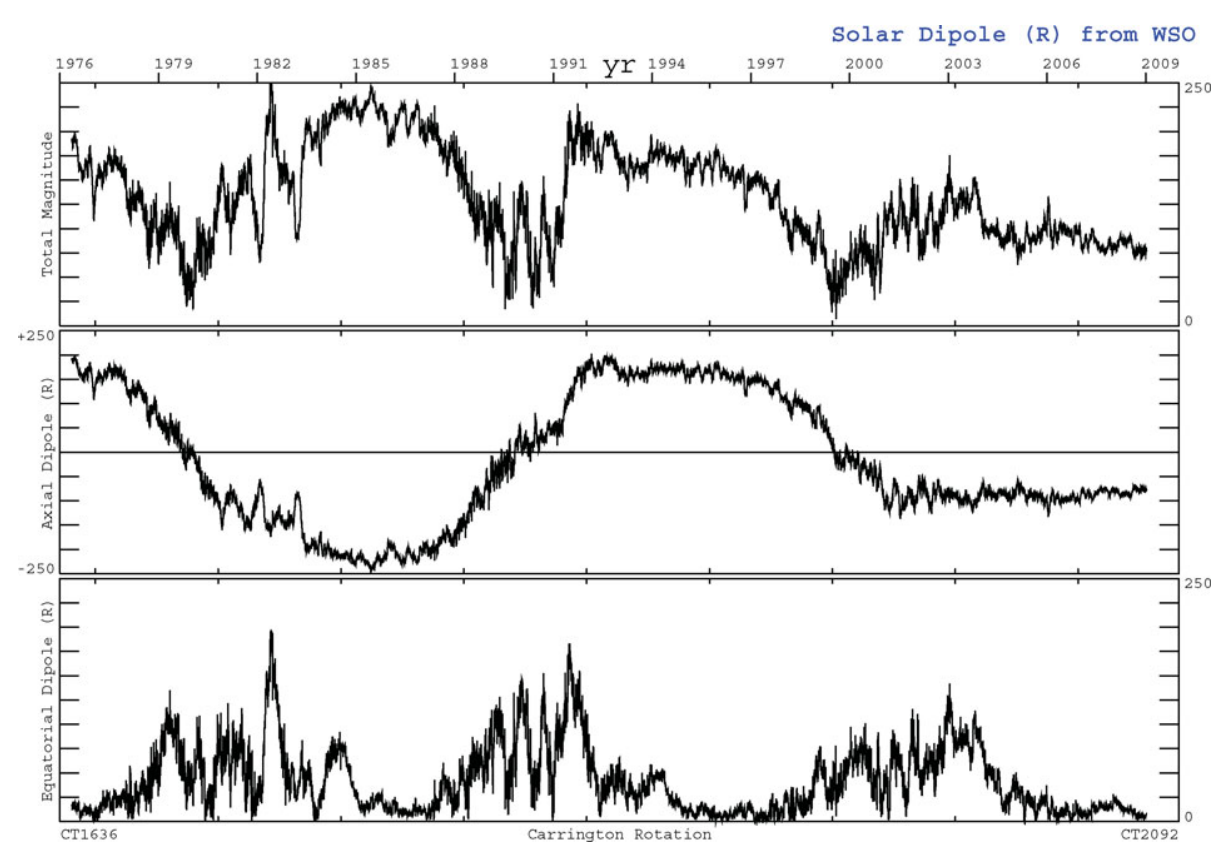

Figure 2. The top panel shows the total magnitude of the solar dipole field from May 1976 to November, 2009, as in Figure 1. The center and bottom panel show its two constituents the axial dipole and the magnitude of the equatorial dipole. The variation is relatively smooth near cycle minima when the axial polar dipole dominates. The dominant feature of the axial dipole is the reversal of the polar field every 11 years about the time of solar maximum. The equatorial dipole is strongly driven by patterns due to the alignment of active regions and their remnants at mid and low latitude. Such alignments have a lifetime of 12-18 months. 
Figure 3 compares the evolution of the source surface field during CYCLES 21 and 23. The left column shows the 10 years of Cycle 21, from 1976 to 1986 . The right column shows 13 years of Cycle 23, from 1996 to 2009. Each panel is two rotations wide to show more clearly the field configuration. The large-scale field usually evolves on a relatively slow time scale, so showing one panel every 12 rotations provides a reasonable view of
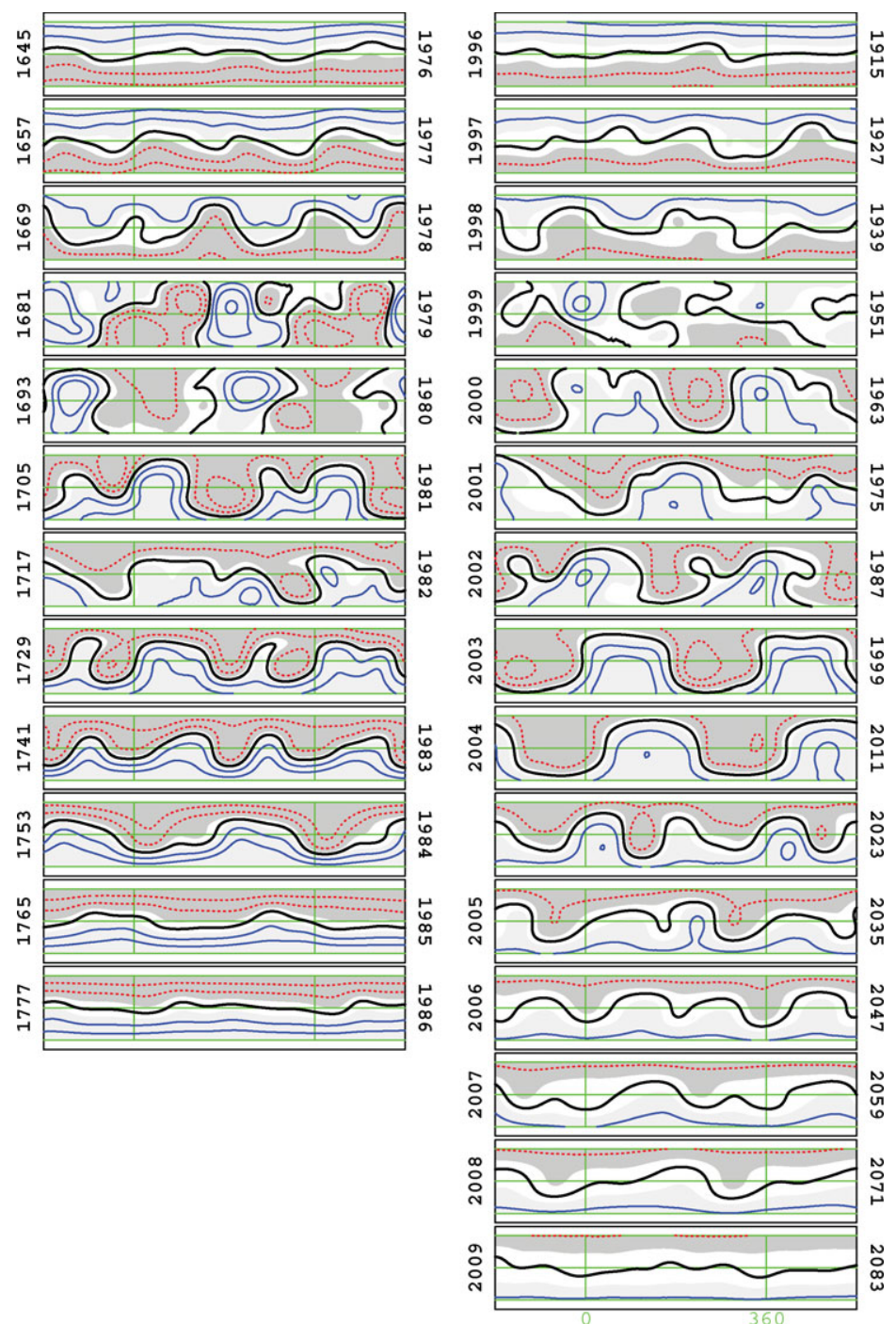

Figure 3. The panels on the left show the Solar Cycle 21 variations of the magnetic field configuration at the base of the solar wind computed with a potential field source surface model. Each panel shows the field at 2.5 solar radii in a two-rotation wide window. The Carrington Rotation is indicated on the left and the year on the right. The vertical axis is sine latitude from pole to pole and data are shown between $70 \mathrm{~N}$ and $70 \mathrm{~S}$. The dark line is the neutral line where the polarity reverses. Light shaded areas with solid contours show positive (out of the Sun) regions. The panels are spaced by 12 rotations, about 11 months, to show the entire 11-year cycle in 12 panels from 1976 to 1986. The panels on the right show the same for Solar Cycle 23. Here the Rotation is indicated on the right and the year on the left, near the center. Cycle 23 lasted longer so more panels are shown, but the polarity was the same as Cycle 21. The neutral line at the base of the heliospheric current sheet is nearly flat at minimum. 
how the field varies. The dominant polar field at solar minimum confines the neutral line to the equator at the beginning and end of each cycle. The Earth, never more than 7 degrees from the equator, encounters the interplanetary extension of the neutral line, the heliospheric current sheet (HCS), every rotation. At higher latitude during long intervals near minimum the polarity of the heliospheric field does not reverse [McComas et al., 2000]. The HCS becomes increasingly distorted during the rise of the cycle as active regions make the photospheric field more complex. As the polar fields weaken in response to old active region flux moving poleward, the HCS crosses all latitudes. Three or four years into each cycle $(1979-1980 ; 1999-2000)$ the polar fields reverse in the corona. This reversal gradually propagates outward to the edge of the heliosphere, affecting the access of cosmic rays. During the declining phase the latitudinal extent of the HCS gradually decreases and the complexity diminishes. Each cycle is unique, but the multi-year stability of the features during the declining phase is quite impressive. Because so little new active region flux emerged during the last few years of Cycle 23, the lifetime of structures is even longer. For example, track the lobe of positive flux near 0 degrees in CR 1987 that drifts slowly to the right through to CR 2059, five years later.

Because the polar field was so weak during Cycle 23, the warps of the HCS remained large for a very long time, well into 2008. In contrast, Cycle 21 (and 22, not shown here) flattened just eight or nine years into the cycle. The coronal holes that are the source of the open flux, also calculable with the PFSS model, also differ late in Cycle 23. The polar coronal holes for the last several years are smaller, and even though the flux at low latitude was small, equatorial coronal holes opened up and contributed significantly to the solar wind. The unipolar caps did not dominate the heliospheric magnetic structure. For several years around the three previous minima virtually no open flux emanated from low or middle latitudes. Note too that Figure 3 shows fewer contours in Cycle 23. This means that with a constant source surface radius, less flux is open to the heliosphere during most of the current cycle. This is qualitatively consistent with the observation of values of open flux in the heliosphere during the current minimum [Smith and Balogh, 2008]. While intriguing, because the height of the source surface is an adjustable and somewhat arbitrary parameter, it is not clear how much significance should be attributed to the details of the agreement. The fact that much of the solar wind observed near Earth at this minimum emerged from smaller near-equatorial holes may explain why solar wind velocity, density, magnitude, and dynamic pressure are all lower.

What is the cause of the weaker polar fields during this minimum?

\section{Zonal Magnetic Fields}

The photospheric field pattern can be explained by surface flux transport acting on the flux that emerges during the cycle [DeVore et al., 1985]. Even though more total flux emerges on the small scale [Zirin, 1987], that flux is not organized and does not contribute significantly to the evolution of the pattern. The magnetic flux distribution in active regions is locally imbalanced systematically because of the differences between leading and following polarity concentrations and because of the inclination specified by Joy's law. Differential rotation, diffusion, and meridional flow act on flux elements to generate the patterns. Much of the emergent flux in active regions cancels locally, but what remains eventually makes its way poleward to form the new polar cap or travels equatorward where it cancels with flux from the other hemisphere.

Considering the entire solar surface, one can investigate the characteristics of flux in each latitude zone during each rotation. Figure 4 shows the zonal magnetic field versus time for the last three solar cycles. The lower panel is the familiar butterfly diagram 
and shows that the total flux is concentrated in the active region belts during most of the cycle. Flux emerges at middle latitude shortly after minimum and gradually moves equatorward. The total polar flux vanishes at solar maximum and is strongest at solar minimum. Cycle 23 is longer and weaker than either Cycle 21 or 22 and there is a long period in 2007 and 2008 where there is very little flux, except in the weak polar regions.

The upper panel shows the net flux. Flux emerges at middle latitudes shortly after minimum, with leading polarity appearing at lower latitude than following polarity. The regions of following polarity expand gradually poleward, cancelling the flux of the old cycle poles and ultimately forming the new polar cap. The surges sometimes move rapidly (in one or two years) from low latitude to high. The surges can be of either polarity, see for example the alternating polarity stripes in the southern hemisphere in 1981 and 1982. When surges reach the poles, the strength of the cap is affected. So, when the leading-polarity positive surge reaches the south pole in late 1990, the polar field reversal temporarily stalls (see Figure 1). Emerging active regions and remnants of decaying spots contribute to these surges. The surges often occur simultaneously in the two hemispheres, but can be symmetric, antisymmetric, or asymmetric about the equator.

The net zonal polarity looks weaker and significantly less organized in Cycle 23. The onset of the cycle looks fairly typical, but the pattern is soon disrupted, with the polarity even at low latitude reversing more than once. This happens in the south in 2002 and in the north in 2003. There seem to be more leading polarity surges, particularly in the south in 1999, 2001, 2002, 2003, and 2005. The north is less clear, but weaker surges of old polarity can be detected in 2000, 2002, and 2004. This apparently has the effect of

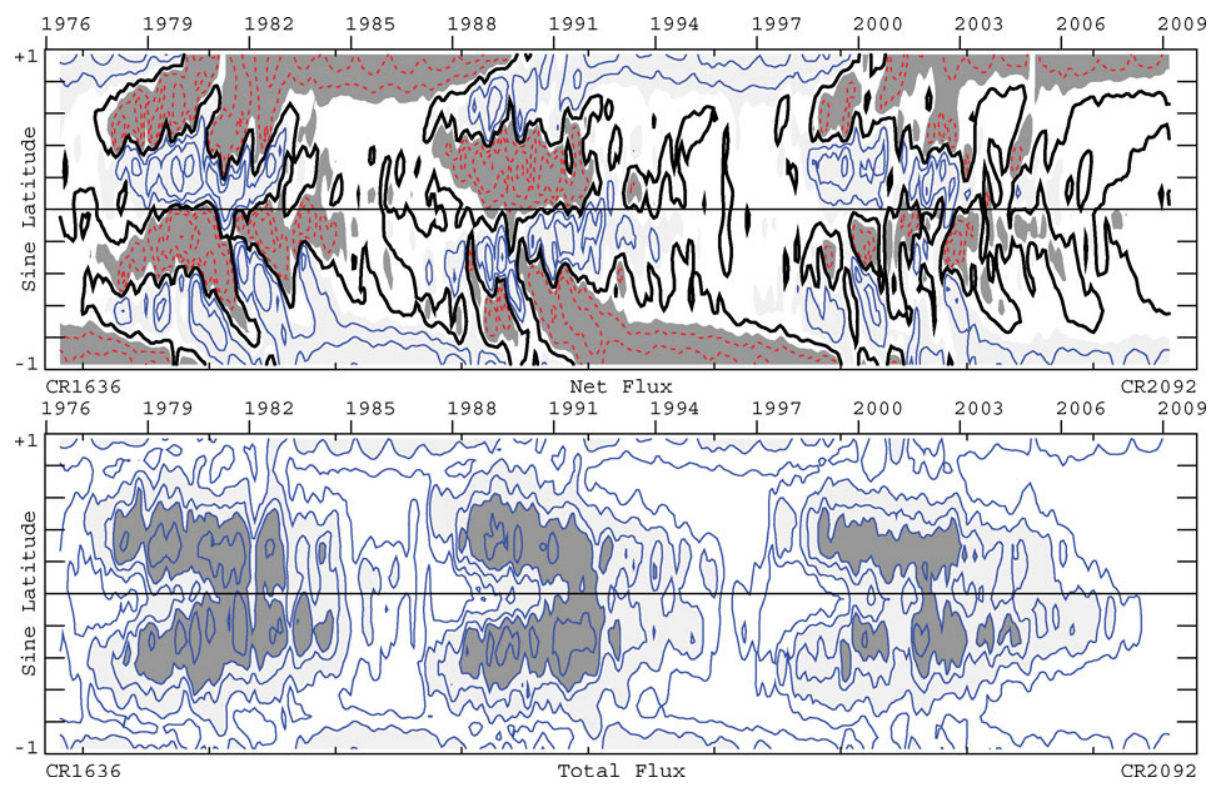

Figure 4. The time evolution of the total flux in each latitude zone is shown in the lower panel for Solar Cycles 21-23. For each Carrington Rotation in each bin in sine latitude the absolute value at each longitude is summed to find the total flux. A contour plot of the result versus time reveals the familiar butterfly diagram. Flux associated with active regions dominates during most of the cycle at lower latitudes. The polar field is strong at higher latitude at other times. The flux emergence moves equatorward through each cycle. The upper panel shows the net flux in each zone, i.e. the sum is performed on the signed values. Structure with the new polar field polarity emerges at mid latitudes shortly after minimum and gradually moves poleward. Each cycle is quite different. 
lowering the polar field strength. This explanation of the weaker polar field obviously begs the question: what about the emergence of active regions led to a different pattern during this cycle?

\section{Discussion}

Long-term observations of the large-scale field are essential to understanding the solar cycle. While the transition from Cycle 23 to 24 is unusual compared to recent cycles, Cycle 14, about 100 years ago, was not that different. Lanza [2009] and others at this meeting show that stars like the Sun have similar dynamo-driven cycles with similar variations in characteristics, but observational limitations make it difficult to be certain of the details. Even solar dynamo models have given very divergent predictions for the magnitude of the upcoming cycle [Pesnell, 2008]. Improved measurements of meridional and other flows in the photospheric and below should help resolve the solar uncertainties. The Kepler and CoRot missions will greatly increase our knowledge of cyclic variations on other stars.

Historically sunspot cycles have overlapped and the current transition is no different, just longer and weaker. Spots from both cycles have been observed from 2007 2009. The curious extended 18-year overlapping solar cycle has not been discussed here, but we can point to three important indications. Altrock [2009] sees the emergence of precursors of the cycle at high latitude in low-coronal Fe XIV emission several years before the minimum in three solar cycles. Torsional oscillations in the upper convection zone also indicate that a zonal flow of enhanced shear appears at high latitude well in advance of the cycle minimum and makes its way equatorward [Howe et al., 2009]. Sunspots eventually emerge at this line of increased shear. Interestingly the equatorward migration is slower during the current declining phase than it was one cycle ago. Lo et al. [2009] have recently extended the analysis of Shrauner and Scherrer [1994] to measure the net large-scale toroidal component of the photospheric field during the last two cycles. The toroidal field is revealed by a slight asymmetry in the magnetic field observed in the east and west hemispheres. The signal is difficult to detect, but also indicates that flux with a reversed toroidal field direction emerges at high latitude well before minimum and gradually migrates equatorward. Future observations and better modeling may help explain why the cycle is extended and overlapping.

\section{References}

Altrock, R. C. 2009, B. A. A. S. 41, 861

Devore, C. R., Sheeley, N. R., Jr., Boris, J. P., Young, T. R., Jr., \& Harvey, K. L. 1985, Solar Physics 102, 41

Hoeksema, J. T., Wilcox, J. M., \& Scherrer, P. H. 1972, J. Geophys. Res. 87, 10331

Howe, R., Christensen-Dalsgaard, J., Hill, F., Komm, R., Schou, J., \& Thompson, M. J. 2009, ApJ. Lett. 701, L87, 10.1088/0004-637X/701/2/L87

Lanza, A. F. 2009, this volume

Lo, L., Hoeksema, J. T., \& Scherrer, P. H. 2009, SOHO-23 Workshop, sun.stanford.edu/ todd/ posters/SOHO23.Lo.3CyclesToroidal.pdf

McComas, D. J. et al. 2000, J. Geophys. Res. 105, 10419.

Pesnell, W. D. 2008, Solar Physics, 252, 209, 10.1007/s11207-008-9252-2.

Schatten, K. 1968, Nature, 220, 1211

Shrauner, J. A. \& Scherrer, P. H. 1994, Solar Physics, 153, 131

Smith, E. J. \& Balogh, A. 2008, Geophys. Res. L. 35, 10.1029/2008GL035345

Svalgaard, L., Duvall, T. L., Jr., \& Scherrer, P. H. 1978, Solar Physics, 58, 255

Zirin, H. 1987, Solar Physics, 110, 101, 10.1007/BF00148205 